\title{
Clinical Impact and Risk Factors of Nonsusceptibility to Third-Generation Cephalosporins Among Hospitalized Adults with Monomicrobial Enterobacteriaceae Bacteremia in Southern Taiwan: A Multicenter Study
}

This article was published in the following Dove Press journal: Infection and Drug Resistance

Tsao-Chin Lin ${ }^{1,2, *}$

Yuan-Pin Hung ${ }^{3,4}$,*

Ching-Chi Lee (iD)

Wei-Tang Lin 6

Li-Chen Huang ${ }^{6}$

Wei Dai ${ }^{7}$

Chi-Shuang Kuo ${ }^{8}$

Wen-Chien Ko iD ${ }^{4,9}$

Yeou-Lih Huang'

'Department of Medical Laboratory and Biotechnology, Kaohsiung Medical

University, Kaohsiung, Taiwan; ${ }^{2}$ Medical

Laboratory, Sinying Hospital, Ministry of

Health and Welfare, Tainan, Taiwan;

${ }^{3}$ Departments of Internal Medicine, Tainan

Hospital, Ministry of Health and Welfare,

Tainan, Taiwan; ${ }^{4}$ Department of Internal

Medicine, National Cheng Kung University

Hospital, College of Medicine, National

Cheng Kung University, Tainan, Taiwan;

${ }^{5}$ Clinical Medicine Research Center.

National Cheng Kung University Hospital,

College of Medicine, National Cheng Kung

University, Tainan, Taiwan; ${ }^{6}$ Medical

Laboratory, Chiayi Hospital, Ministry of

Health and Welfare, Chiayi, Taiwan;

${ }^{7}$ Experiment and Diagnosis, Tainan Hospital,

Ministry of Health and Welfare, Tainan,

Taiwan; ${ }^{8}$ Medical Laboratory, Pingtung

Hospital, Ministry of Health and Welfare,

Pingtung, Taiwan; ${ }^{9}$ Department of Medicine,

College of Medicine, National Cheng Kung

University, Tainan, Taiwan

*These authors contributed equally to this work

Correspondence: Yeou-Lih Huang Department of Medical Laboratory and Biotechnology, Kaohsiung Medical

University, No. 100, Shin-Chuan Ist Road,

Sanmin Dist., Kaohsiung, 80708, Taiwan

Tel +8867312110 I Ext. 2273

Fax +88673113449

Email yelihu@kmu.edu.tw
Background: Reducing the effectiveness of broad-spectrum cephalosporins against Enterobacteriaceae infections has been recognized. This study aimed to investigate risk factors and clinical significance of third-generation cephalosporin nonsusceptibility (3GCNS) among the cases of monomicrobial Enterobacteriaceae bacteremia $(\mathrm{mEB})$ at regional or district hospitals.

Methods: The study was conducted at three hospitals in southern Taiwan between Jan. 2017 and Oct. 2019. Only the first episode of $\mathrm{mEB}$ from each adult (aged $\geq 20$ years) was included. The primary outcome was in-hospital crude mortality.

Results: Overall there were 499 episodes of adults with mEB included, and their mean age was 74.5 years. Female predominated, accounting for $53 \%$ of all patients. Escherichia coli $(62 \%)$ and Klebsiella pneumoniae $(21 \%)$ were two major causative species. The overall mortality rate was $15 \%$ (73/499), and patients infected by 3 GC-NS isolates $(34 \%, 172 / 499)$ had a higher mortality rate than those by $3 \mathrm{GC}$-susceptible isolates $(66 \%, 327 / 499)(21 \% \mathrm{vs}$ $11 \%, P=0.005)$. By the multivariate analysis, 3GC-NS was the only independent prognostic determinant (adjusted odds ratio [AOR], 1.78; $P=0.04$ ). Of note, male (AOR 2.02, $P=0.001$ ), nosocomial-acquired bacteremia (AOR 2.77, $P<0.001$ ), and usage of nasogastric tube (AOR 2.01, $P=0.002$ ) were positively associated with $3 \mathrm{GC}-\mathrm{NS}$, but $P$. mirabilis bacteremia (AOR $0.28, P=0.01$ ) and age (AOR $0.98, P=0.04$ ) negatively with $3 \mathrm{GC}-\mathrm{NS}$.

Conclusion: For adults with Enterobacteriaceae bacteremia, 3GC-NS signifies a significant prognostic impact. Efforts to rapid identification of such antimicrobial resistance profiles should be incorporated into antimicrobial stewardship programs to achieve favorable outcomes

Keywords: third-generation cephalosporin, nonsusceptible, Enterobacteriaceae, Klebsiella pneumoniae, male, nasogastric tube

\section{Introduction}

Enterobacteriaceae isolates are responsible for a wide variety of nosocomial and community-acquired infections and third-generation cephalosporins (3GCs) are administered as the main choice for the treatment of infections caused by these microorganisms. $^{1-7}$ However, along with the over-prescription of $3 \mathrm{GCs}$ by 
clinicians, reducing the therapeutic efficacy of these antimicrobial agents for Enterobacteriaceae infections ${ }^{1,7-11}$ and the increasing trend in nonsusceptibility (NS) to 3GCs were recently evidenced. ${ }^{12}$ Moreover, infections caused by 3GC-resistant Enterobacteriaceae were significantly associated with the increasing hazard of death and excess length of stay and costs. ${ }^{13}$ Taking Escherichia coli as an example, the total cost attributable to excess hospital stays for bloodstream infections caused by 3GC-resistant isolates was estimated up to 18.1 million Euros each year in Europe. ${ }^{14}$ Furthermore, for patients with Enterobacter bacteremia, the 30-day mortality rate of patients infected by 3GC-resistant isolates was significantly higher than those by $3 \mathrm{GC}$-susceptible isolates, regardless of whether stratified by infection sites or by the initial presence of septic shock. ${ }^{15}$

In a medical center in northern Taiwan, the overall proportion of $3 \mathrm{GC}$ resistance in community-onset $E$. coli bacteremia has been up to $19.7 \% .{ }^{16}$ Nevertheless, the clinical impact of $3 \mathrm{GC}$ resistance on the prognoses of patients with Enterobacteriaceae bacteremia in the district or regional hospitals in Taiwan was not reported yet. Accordingly, the aim of the present multicenter study was to investigate risk factors of 3GC-NS Enterobacteriaceae bacteremia and their adverse influence on outcomes.

\section{Methods}

\section{Study Design and Population}

The study was conducted at three hospitals of the Ministry of Health and Welfare in southern Taiwan: Tainan Hospital (A, a 300-bed district hospital), Sinying Hospital (B, a 78bed regional hospital), and Chiayi Hospital (C, a 237-bed regional hospital). There were infection-disease specialists dealing with antibiotic stewardship program at the study hospitals. The study periods spanned between Jan. 1, 2017 and Oct. 31, 2019. The episodes of monomicrobial Enterobacteriaceae bacteremia $(\mathrm{mEB})$ in hospitalized adults (aged $\geq 20$ years) were analyzed, and only the first episode in each patient was included during the study period. The study was reviewed and approved by the Institutional Review Board of National Cheng Kung University Hospital (A-ER-105-183).

\section{Data Collection}

By reviewing the electronic medical charts, a predetermined form was adapted to collect clinical characters, in terms of patient gender, age, hospitalization duration, usage of vasopressor agents and antimicrobials, types and severity (Charlson comorbidity index) of comorbidities, laboratory data, and clinical outcomes. The primary outcome was the in-hospital crude mortality after bacteremia onset. Patients were excluded if they had incomplete clinical information.

\section{Microbiological Methods}

Blood cultures were processed in the BD BACTEC 9240 system (Becton Dickinson, USA). Bacterial species were identified by the morphology and color in the chromogenic agar, and confirmed by BD GNB ID or BD E/NF crystal kit (Becton Dickinson, USA). Antibiotic susceptibility was determined by the disk diffusion method, in accordance with the procedures of the Clinical and Laboratory Standards Institute (CLSI), and was interpreted according to the zone criteria of CLSI issued in 2018 (M100-S21). ${ }^{17}$ The drugs tested included ampicillin, ampicillin/sulbactam, gentamicin, amikacin, cefazolin, cefuroxime, ceftriaxone, ceftazidime, cefepime, ciprofloxacin, ertapenem, and imipenem or meropenem. Susceptibility to 3GCs was defined as the clear zone diameter of ceftriaxone $\geq 23 \mathrm{~mm}$, cefotaxime $\geq 26 \mathrm{~mm}$, or ceftazidime $\geq 21 \mathrm{~mm},{ }^{17}$ and susceptibility to fluoroquinolones as the clear zone diameter of levofloxacin $\geq 17 \mathrm{~mm}$ or ciprofloxacin $\geq 21 \mathrm{~mm}^{17}$

\section{Definitions}

Bacteremia was defined as bacterial growth of blood cultures drawn from central or peripheral venipuncture. As previously described, ${ }^{18,19}$ the administration of antimicrobial therapy was considered to be appropriate when all the following criteria were fulfilled: (i) the administrated antimicrobial was in vitro active against causative microorganisms isolated from blood cultures, based on the contemporary CLSI breakpoints. ${ }^{17}$ (ii) the route and dosage of antimicrobials were administered as recommended in accordance with the Sanford Guide to Antimicrobial Therapy 2018. ${ }^{20}$ As per the previous definition, ${ }^{21}$ antimicrobials administered within 3 days after bacteremia onset were regarded as empirical therapy, and those administered after 3 days of onset when the identification and susceptibility data of bacteremic isolates were available were referred to as definitive therapy. Nosocomial bacteremia was defined as the onset of bacteremia occurring at $\geq 48$ hours after admission. ${ }^{22}$

Septic shock was defined as a mean arterial pressure of $<75 \mathrm{mmHg}$ and usage of vasopressor administration. ${ }^{23}$ Comorbidities were defined as described previously. ${ }^{24}$ 
Malignancies included hematological malignancies and solid tumors. The severity of preexisting medical diseases was assessed by a previously delineated classification system (Charlson comorbidity index). ${ }^{25}$ Crude mortality was defined as death from all causes.

\section{Statistical Analysis}

Statistical analysis was performed by the statistical software (SPSS, version 13.0). Descriptive statistics, including the means, standard deviations, and ranges, were used to analyze the continuous variables. For categorical variables, the percentages and confidence intervals were used. The independent- $t$-test was applied for the continuous variables and the chi-square test or Fisher's exact test for the categorical variables. To identify the predictors and impact of 3GC NS, the variables with a $P$ value less than 0.1 recognized by the univariate analysis were processed by a stepwise, backward logistic regression model. A two-tailed $P$ value of less than 0.05 was considered to be statistically significant.

\section{Results}

\section{Patient Demographics}

After excluding recurrent episodes and polymicrobial bacteremia, there were 293, 88, and 118 episodes of $\mathrm{mEB}$ in the hospital A, B and C, respectively, were included for the analysis (Figure 1). The crude in-hospital mortality rate was similar in three hospitals: $15 \%, 16 \%$, and $13 \%$, respectively. Overall, this study involved 499 adults with an average age of 75 years and their crude in-hospital mortality rate was $15 \%$. Female gender $(263,53 \%)$ predominated. Common comorbidities of the included patients included hypertension, diabetes mellitus, old stroke, chronic kidney diseases, and malignancy, and in this study major microorganisms causing mEB were E. coli, Klebsiella pneumoniae, Proteus mirabilis, Providencia stuartii, and Citrobacter koseri (Table 1).

\section{Risk Factors of In-Hospital Crude Mortality}

As compared with the survivors, fatal patients with $\mathrm{mEB}$ were less likely to be infected by $3 \mathrm{GC}$ - $(68 \%$ vs $51 \%$, $P=0.005)$ or ertapenem-susceptible isolates (95\% vs $85 \%$, $P=0.009$ ) and $E$. coli (65\% vs 48\%, $P=0.008$ ) (Table 1), but were often associated with $K$. pneumoniae infections (19\% vs $33 \%, P=0.008$ ). No significant association between the inhospital mortality and patient age, gender male, comorbidity types or Charlson comorbidity index was disclosed. Notably, in the multivariate analysis for risk factors of in-hospital crude mortality, only one independent variable, 3GC-NS, was recognized (adjusted odds ratio [AOR], 1.78; 95\% confident interval $[\mathrm{CI}] 1.02-3.11 ; P=0.04$ ) (Table 2).

\section{Predictors of 3GC-Nonsusceptibility Among Enterobacteriaceae Bacteremia}

Patients infected by 3GC-NS isolates were older (mean age: 77 years vs 74 years; $\mathrm{P}=0.009$ ) and more likely to be male gender ( $58 \%$ vs $42 \%, P<0.001)$, or to have comorbidities of chronic kidney diseases ( $23 \%$ vs $12 \%, P=0.003$ ) or the use of nasogastric tubes $(61 \%$ vs $37 \%, P<0.001)$ or urinary catheters $(55 \%$ vs $38 \%, P<0.001)$ than those by 3GC-susceptible microorganisms, as shown in Table 3. Otherwise, less episodes of $P$. mirabilis bacteremia $(3 \%$ vs $8 \%, P=0.03)$ and more $K$. pneumonia bacteremia $(30 \%$ vs $16 \%, P<0.001)$ were noted in patients with $3 \mathrm{GC}-\mathrm{NS}$ Enterobacteriaceae bacteremia (Table 3). Since chronic kidney disease was the only parameter in Charlson comorbidity index with statistically correlated with 3GC-NS, chronic kidney disease, instead of Charlson comorbidity index, was placed in the multivariate analysis. In the multivariate analysis, male patients (AOR 2.02, 95\% CI 1.33-3.05; $P=0.001)$, nosocomial-acquired bacteremia (AOR 2.77, 95\% CI 1.72-4.47; $P<0.001$ ), and usage of nasogastric tube (AOR 2.01, 95\% CI 1.28-3.16; $P=0.002$ ) were positively associated with $3 \mathrm{GC}-\mathrm{NS}$ (Table 4). In contrast, P. mirabilis bacteremic episodes (AOR 0.28, 95\% CI $0.10-0.77 ; P=0.01$ ) and age (AOR 0.98, 95\% CI $0.97-0.99 ; P=0.04)$ were negatively linked to $3 \mathrm{GC}-\mathrm{NS}$.

\section{Antimicrobial Therapy and Clinical Outcomes}

The common antimicrobials empirically administered for patients with 3GC-susceptible Enterobacteriaceae bacteremia were $3 \mathrm{GCs}(32 \%), 2 \mathrm{GCs}(16 \%)$, and piperacillintazobactam (14\%). Appropriate empirical (30\%vs.82\%, $P<0.001)$ or definitive $(80 \%$ vs.94\%, $P<0.001)$ therapy was less commonly prescribed among patients infected by $3 \mathrm{GC}-\mathrm{NS}$ isolates, compared to those by $3 \mathrm{GC}$ susceptible isolates (Table 5). Furthermore, patients with 3GC-NS Enterobacteriaceae bacteremia more often had a septic shock at presentation $(20 \%$ vs. $11 \%, P=0.007)$ and had a higher in-hospital crude mortality rate $(21 \%$ vs $11 \%$, $P=0.005)$ than those infected by $3 \mathrm{GC}$-susceptible isolates (Table 5). 

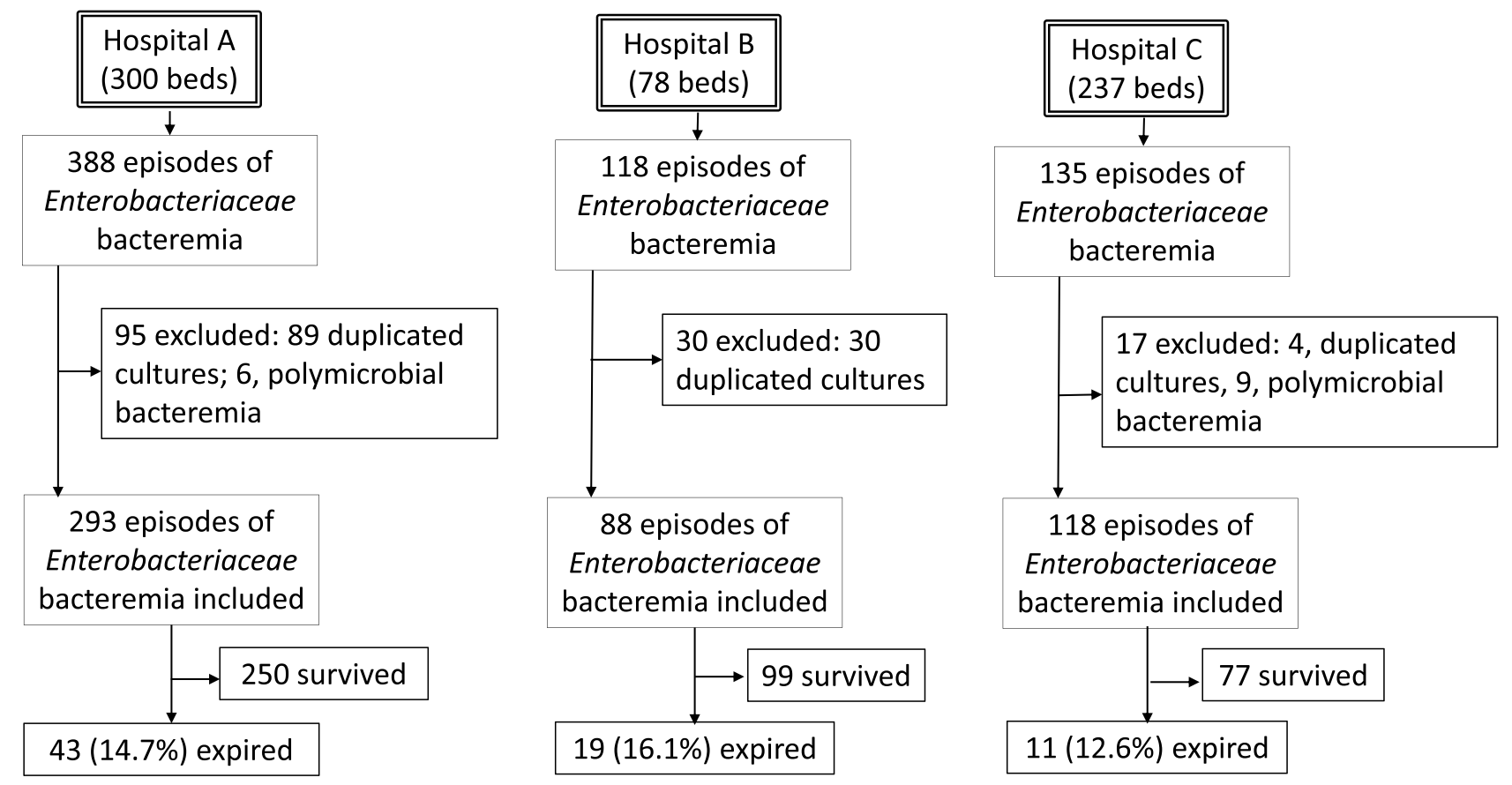

Figure I A flowchart of patient selection in three study hospitals.

\section{Discussion}

Patients with 3GC-NS mEB were associated with longer hospital or ICU stays and a worse outcome. However, such a result needs to be interpreted with caution, because patients infected by the isolates of antimicrobial-resistant Enterobacteriaceae were older and had more comorbidities. ${ }^{1,26}$ Moreover, the other explanation for unfavorable prognoses might result from delayed administration of appropriate antibiotics. ${ }^{18,19}$ Similar to the previous report, ${ }^{1}$ inadequate empirical antibiotic therapy was more common in patients with $3 \mathrm{GC}-\mathrm{NS}$ mEB than in those with bacteremia due to 3GC-susceptible Enterobacteriaceae isolates.

In our study, male patients, nosocomial-acquired bacteremia, and usage of nasogastric tube were positively associated with 3GC-NS; and in contrast, P. mirabilis bacteremic episodes and age were negatively linked to $3 \mathrm{GC}$ NS. Factors correlating to the acquisition of Enterobacteriaceae strains harboring $3 \mathrm{GC}$ resistance have been identified before, including male gender, ${ }^{1}$ prior exposure to antimicrobial agents, $1,16,26$ underlying disease, ${ }^{1,16,26}$ indwelling device or prosthesis, ${ }^{16,26}$ or surgery. ${ }^{26}$ Likewise, several variables, including male gender, age, usage of nasogastric tube, and bacteremic episodes due to $K$. pneumoniae, were independently linked to $3 \mathrm{GC}-\mathrm{NS} \mathrm{mEB}$ in our cohort. However, some predictors identified in previous investigations, ${ }^{1,16,26}$ such as the presence of an invasive prosthesis or intravascular catheter, recent surgery or hospitalization, or residence in nursing home or long-term care facility were not assessed in the present retrospective study.

The ratio of appropriate empirical therapy was lower among patients infected by either 3GC-NS (30\%) or $3 \mathrm{GC}$-susceptible isolates $(82 \%)$ in our study. In a retrospective Dutch study of bacteremia episodes caused by 3GC-resistant and 3GC-sensitive Enterobacteriaceae bacteremia in 2015, 56\% and 94\% were empirically treated with appropriate antibiotics, respectively. ${ }^{27}$ Nevertheless, the $3 \mathrm{GC}$-resistant rate in the former study was $8.3 \%(64 / 773),{ }^{27}$ much lower than the $3 \mathrm{GC}-\mathrm{NS}$ rate of $34.5 \%(172 / 499)$ in our study. The higher nonsusceptible rates toward antimicrobial agents among Enterobacteriaceae bacteremia isolates in recent years led clinicians more difficulty in selecting appropriate empirical antimicrobial agents, especially for extended-spectrum $\quad \beta$-lactamase $\quad$ (ESBL)-producing bacteria. ${ }^{28}$ Though ESBL-producing phenotype was not examined, the high $3 \mathrm{GC}-\mathrm{NS}$ rate among our Enterobacteriaceae isolates might partly be contributed by the presence of ESBL-producing isolates. In the era of reduced susceptibility to 3GCs among Enterobacteriaceae isolates, the information of the clinical parameters predictive of 3GC-NS identified in our study is helpful to select appropriate antimicrobial agents. 
Table I Factors Associated with In-Hospital Crude Mortality in Patients with Monomicrobial Enterobacteriaceae Bacteremia

\begin{tabular}{|c|c|c|c|c|}
\hline \multirow[t]{2}{*}{ Variables } & \multirow{2}{*}{$\begin{array}{l}\text { Total } \\
\mathrm{n}=499\end{array}$} & \multicolumn{2}{|c|}{ Patient Numbers (\%) } & \multirow[t]{2}{*}{$P$ value } \\
\hline & & Survived, $n=426$ & Expired, $n=73$ & \\
\hline Age, years, mean $\pm S D$ & $74.5 \pm 12.9$ & $74.1 \pm 13.0$ & $77.3 \pm 11.5$ & 0.05 \\
\hline Gender, male & $236(47.3)$ & $20 \mathrm{I}(47.2)$ & $35(47.9)$ & 1.00 \\
\hline \multicolumn{5}{|l|}{ Comorbidities } \\
\hline Hypertension & $286(57.3)$ & $246(57.7)$ & $40(54.8)$ & 0.70 \\
\hline Diabetes mellitus & $207(4 I .5)$ & $177(4 \mid .5)$ & $30(4 I .1)$ & 1.00 \\
\hline Old stroke & $105(21.0)$ & $86(20.2)$ & $19(26.0)$ & 0.28 \\
\hline Chronic kidney diseases & $79(15.8)$ & $64(15.0)$ & $15(20.5)$ & 0.23 \\
\hline Malignancy & $66(13.2)$ & $54(12.7)$ & $12(16.4)$ & 0.36 \\
\hline Coronary artery disease history & $58(11.6)$ & $48(11.3)$ & $10(13.7)$ & 0.55 \\
\hline Liver cirrhosis & $22(4.4)$ & $18(4.2)$ & $4(5.5)$ & 0.55 \\
\hline Congestive heart failure & $14(2.8)$ & $13(3.1)$ & I (I.4) & 0.70 \\
\hline Charlson comorbidity index, mean \pm SD & $1.2 \pm 1.2$ & $1.2 \pm 1.1$ & $1.4 \pm 1.3$ & 0.15 \\
\hline Nosocomial bacteremia & $112(22.4)$ & $91(21.4)$ & $21(28.8)$ & 0.17 \\
\hline Time-to-positivity, hours & $25.1 \pm 17.4$ & $24.5 \pm 17.4$ & $28.5 \pm 19.4$ & 0.10 \\
\hline \multicolumn{5}{|l|}{ Antimicrobial-susceptible isolates } \\
\hline Second GCs & $223(44.7)$ & $197(46.2)$ & $26(35.6)$ & 0.10 \\
\hline Third GCs & $327(65.5)$ & $290(68.1)$ & $37(50.7)$ & 0.005 \\
\hline Fourth GCs & $363(72.7)$ & $316(74.2)$ & $47(64.4)$ & 0.09 \\
\hline Amoxicillin/clavulanic acid & $297(57.5)$ & $250(58.7)$ & $37(50.7)$ & 0.20 \\
\hline Fluoroquinolones & $259(51.9)$ & $224(52.6)$ & $35(47.9)$ & 0.53 \\
\hline Ertapenem & $465(93.2)$ & $403(94.6)$ & $62(84.9)$ & 0.009 \\
\hline \multicolumn{5}{|l|}{ Major causative microorganisms } \\
\hline Escherichia coli & $310(62.1)$ & $275(64.6)$ & $35(47.9)$ & 0.009 \\
\hline Klebsiella pneumoniae & $104(20.8)$ & $80(18.8)$ & $24(32.9)$ & 0.008 \\
\hline Proteus mirabilis & $31(6.2)$ & $27(6.3)$ & $4(5.5)$ & 1.00 \\
\hline Providencia stuartii & II (2.2) & $10(2.3)$ & I (I.4) & 1.00 \\
\hline Citrobacter koseri & $8(1.6)$ & $7(1.6)$ & I (I.4) & 1.00 \\
\hline
\end{tabular}

Notes: Data are given as number (percent), unless otherwise specified. Boldface indicates statistical significance in the univariate analysis, ie, a $P$ value of $<0.05$. Abbreviations: GC, generation cephalosporin; SD, standard deviation.

The rapid emergence of drug-resistant strains and novel viruses has motivated the search for new anti-infectious agents. In recent years, a reexamination of traditional medicines has become more common and has already provided several new antibiotics. Some new clinical therapies, such as pump inhibitors in association with common antibiotic therapies and natural molecules such as oils essential are already clinically available. For example, Usai D and other scholars have shown that novel antibiotics with new modes of action are urgently required to suppress the rise of MDR 
Table 2 Multivariate Analysis of Risk Factors for In-Hospital Crude Mortality Among Adults with Monomicrobial Enterobacteriaceae Bacteremia

\begin{tabular}{|l|c|c|c|}
\hline Characters & Adjusted Odds Ratio & 95\% Confidence Interval & P value \\
\hline 3GC nonsusceptibility & $\mathbf{1 . 7 8}$ & $\mathbf{I . 0 2 - 3 . 1 1}$ & $\mathbf{0 . 0 4}$ \\
\hline Ertapenem nonsusceptibility & 1.55 & $0.61-3.91$ & 0.36 \\
\hline Klebsiella pneumoniae & 1.12 & $0.51-2.49$ & 0.78 \\
\hline Escherichia coli & 0.60 & $0.31-1.19$ & 0.14 \\
\hline
\end{tabular}

Note: Boldface indicates statistical significance in the univariate analysis, ie, a $P$ value of $<0.05$.

Abbreviation: 3GC, third-generation cephalosporin.

Table 3 Clinical Predictors of Third-Generation Cephalosporin Nonsusceptibility in the Episodes of Monomicrobial Enterobacteriaceae Bacteremia

\begin{tabular}{|c|c|c|c|}
\hline \multirow[t]{2}{*}{ Variables } & \multicolumn{2}{|c|}{ Patient Numbers (\%) } & \multirow[t]{2}{*}{$P$ value } \\
\hline & Susceptible, $n=327$ & Nonsusceptible, $n=172$ & \\
\hline Age, years, mean \pm SD & $73.5 \pm 13.0$ & $76.6 \pm 12.3$ & 0.009 \\
\hline Charlson comorbidity index, mean \pm SD & $\mathbf{I . I} \pm \mathrm{I.I}$ & $1.4 \pm 1.2$ & 0.007 \\
\hline Gender, male & $137(41.9)$ & $99(57.6)$ & $<0.001$ \\
\hline \multicolumn{4}{|l|}{ Comorbidities } \\
\hline Hypertension & $190(58.1)$ & $96(55.8)$ & 0.64 \\
\hline Diabetes mellitus & $133(40.7)$ & $74(43.0)$ & 0.63 \\
\hline Old stroke & $68(20.8)$ & $37(21.5)$ & 0.91 \\
\hline Chronic kidney disease & $40(12.2)$ & $39(22.7)$ & 0.003 \\
\hline Malignancy & $43(13.1)$ & $23(13.4)$ & 1.00 \\
\hline Coronary artery disease & $32(9.8)$ & $26(15.1)$ & 0.08 \\
\hline Liver cirrhosis & $12(3.7)$ & $10(5.8)$ & 0.26 \\
\hline Congestive heart failure & $8(2.4)$ & $6(3.5)$ & 0.57 \\
\hline Nosocomial bacteremia & 48 (14.7) & $64(37.2)$ & $<0.001$ \\
\hline \multicolumn{4}{|l|}{ Catheter dependence } \\
\hline Nasogastric tube & $120(36.9)$ & $104(60.8)$ & $<0.001$ \\
\hline Urinary catheter & $124(37.9)$ & $94(55.3)$ & $<0.001$ \\
\hline \multicolumn{4}{|l|}{ Major causative microorganisms } \\
\hline Escherichia coli & $210(64.2)$ & $100(58.1)$ & 0.21 \\
\hline Klebsiella pneumoniae & $5 I(15.6)$ & $52(30.2)$ & $<0.001$ \\
\hline Proteus mirabilis & $26(7.9)$ & $5(2.9)$ & 0.03 \\
\hline Providencia stuartii & $10(3.1)$ & I (0.6) & 0.11 \\
\hline Citrobacter koseri & $6(1.8)$ & $2(1.2)$ & 0.72 \\
\hline
\end{tabular}

Notes: Data are given as number (percent), unless otherwise specified. Boldface indicates statistical significance in the univariate analysis, ie, a $P$ value of $<0.05$. Abbreviation: SD, standard deviation. 
Table 4 Multivariate Analysis of Risk Factors of Third-Generation Cephalosporin Nonsusceptibility Among the Episodes of Monomicrobial Enterobacteriaceae Bacteremia

\begin{tabular}{|c|c|c|c|}
\hline Characters & Adjusted Odds Ratio & 95\% Confidence Interval & $P$ value \\
\hline \multicolumn{4}{|l|}{ Patient demographics } \\
\hline Male & 2.02 & $1.33-3.05$ & 0.001 \\
\hline Age, years & 0.98 & $0.97-0.99$ & 0.04 \\
\hline Nosocomial bacteremia & 2.77 & $1.72-4.47$ & $<0.001$ \\
\hline \multicolumn{4}{|l|}{ Catheter dependence } \\
\hline Nasogastric tubes & 2.01 & $1.28-3.16$ & 0.002 \\
\hline Urinary catheters & 1.39 & $0.89-2.18$ & 0.15 \\
\hline \multicolumn{4}{|l|}{ Causative microorganisms } \\
\hline Klebsiella pneumoniae & 1.48 & $0.90-2.45$ & 0.13 \\
\hline Proteus mirabilis & 0.28 & $0.10-0.77$ & 0.01 \\
\hline Underlying chronic kidney diseases & 1.58 & $0.93-2.69$ & 0.09 \\
\hline
\end{tabular}

Note: Boldface indicates statistical significance in the univariate analysis, ie, a $P$ value of $<0.05$.

Table 5 Bacteremia Severity, Antimicrobial Therapy and Outcomes of Patients with Monomicrobial Enterobacteriaceae Bacteremia, Stratified by Third-Generation Cephalosporin Susceptibility

\begin{tabular}{|c|c|c|c|}
\hline \multirow[t]{2}{*}{ Variables } & \multicolumn{2}{|c|}{ Patient Numbers (\%) } & \multirow[t]{2}{*}{$P$ value } \\
\hline & Susceptible, $n=327$ & Nonsusceptible, $n=172$ & \\
\hline \multicolumn{4}{|l|}{ Bacteremia severity } \\
\hline Blood leukocyte, $\times 10^{3} / \mathrm{mm}^{3}$, mean $\pm \mathrm{SD}$ & $13.3 \pm 7.4$ & $13.0 \pm 6.0$ & 0.72 \\
\hline Time-to-positivity, hours, mean \pm SD $(n=392)$ & $24.5 \pm 16.6$ & $26.1 \pm 19.5$ & 0.39 \\
\hline Initial presentation of septic shock & $36(11.0)$ & $35(20.3)$ & 0.007 \\
\hline Requirement of intensive care & $61(18.7)$ & $33(19.2)$ & 0.90 \\
\hline \multicolumn{4}{|l|}{ Appropriate antimicrobial therapy } \\
\hline Empirical & $267(81.7)$ & $52(30.2)$ & $<0.001$ \\
\hline Definitive & 307 (93.9) & $138(80.2)$ & $<0.001$ \\
\hline \multicolumn{4}{|l|}{ Outcomes } \\
\hline Length of hospitalization, days, mean $\pm S D$ & $20.2 \pm 58.1$ & $26.9 \pm 41.3$ & 0.15 \\
\hline In-hospital crude mortality & $37(11.3)$ & $36(20.9)$ & 0.005 \\
\hline
\end{tabular}

Notes: Data are given as number (percent), unless otherwise specified. Boldface indicates statistical significance under the univariate analysis, ie, a $P$ value of $<0.05$. Abbreviation: SD, standard deviation.

bacteria. An alternative approach would be to identify molecules that can interfere with the process of efflux. ${ }^{29}$ In 2006, researchers discovered that the region around amino acid Val-610 in YhiV appears to be involved in determining recognition and efficiency of export of a number of MDR efflux pump substrates. This single point mutation in the periplasmic loop of the pump can increase resistance to a given drug such as a fluoroquinolone while decreasing resistance to another one. ${ }^{30}$ Moreover, South African scholars have shown that in vitro potentiation of carbapenems with tannic acid against carbapenemase-producing enterobacteriaceae. ${ }^{31}$ 
In addition, antiviral activities of essential oils from the leaves, rhizomes, and whole plant of Hornstedtiabella were investigated, the GC/MS analysis showed that $\beta$-pinene, E- $\beta$ caryophyllene, and $\alpha$-humulene were found at high concentrations in the essential oils. ${ }^{32}$ Other researchers discovered that Biological activities of essential oil extracted from leaves of Atalantiasessiflora and Limnocitrus littoralis that showed antimicrobial activities against Gram-positive strains as Staphylococcus; Gram-negative bacteria such as Klebsiella pneumoniae and Escherichia coli. ${ }^{33,34}$ Furthermore, American scholars have pointed out that in vitro activity of essential oils against Gram-positive and Gram-negative clinical isolates, including Carbapenem-Resistant Enterobacteriaceae. ${ }^{35}$ Traditional medicine plants are likely to provide further new antibiotics in the future. However, the use of plant extracts or pure natural compounds in combination with conventional antibiotics may hold greater promise for rapidly providing affordable treatment options.

There were several limitations in this study. First, though there were only nearly 500 cases of $\mathrm{mEB}$ included in the cohort, it is a multicenter study conducted at three district and regional hospitals in southern Taiwan, representing the population other than the patients cared for at medical centers. Second, recall biases due to the retrospective nature of study design unavoidably exist, and the number of factors related to 3GC NS are likely to be underestimated. Third, not all 3GCs (such as cefotaxime, ceftriaxone, and ceftazidime) were tested for antimicrobial susceptibility. Overestimating or underestimating 3GC-NS among the included Enterobacteriaceae isolates is not known, and the study results should be interpreted cautiously. Last, other clinical variables, such as the degree or timing of source control, or the dosage or regimens of antimicrobial therapy were assessed as the potential ones affecting the prognosis of the cases of $\mathrm{mEB}$.

\section{Conclusion}

In conclusion, the presence of $3 \mathrm{GC}-\mathrm{NS}$ in the etiological pathogens of $\mathrm{mEB}$ is independently correlated with a poor prognosis. Rapid identification of antimicrobial resistance by clinical predictors or new methods of susceptibility testing shall be incorporated in antimicrobial stewardship programs to improve patient outcomes.

\section{Ethics Approval and Consent to Participate}

The study was reviewed and approved by the Institutional Review Board of National Cheng Kung University
Hospital in accordance with the Declaration of Helsinki. Informed consent was waived because of the retrospective nature of the study and the analysis used anonymous clinical data.

\section{Acknowledgments}

This work was supported by the Ministry of Health and Welfare, Taiwan.

\section{Disclosure}

The authors report no conflicts of interest in this work.

\section{References}

1. Oliveira MC, Oliveira CR, Goncalves KV, Santos MS, Tardelli AC, Nobre VA Jr. Enterobacteriaceae resistant to third generation cephalosporins upon hospital admission: risk factors and clinical outcomes. Braz J Infect Dis. 2015;19(3):239-245. doi:10.1016/j. bjid.2015.01.006

2. Liu LH, Wang NY, Wu AY, Lin CC, Lee CM, Liu CP. Citrobacter freundii bacteremia: risk factors of mortality and prevalence of resistance genes. J Microbiol Immunol Infect. 2018;51(4):565-572. doi:10.1016/j.jmii.2016.08.016

3. Syue LS, Tang HJ, Hung YP, et al. Bloodstream infections in hospitalized adults with dengue fever: clinical characteristics and recommended empirical therapy. J Microbiol Immunol Infect. 2019;52 (2):225-232. doi:10.1016/j.jmii.2018.11.003

4. Erlanger D, Assous MV, Wiener-Well Y, Yinnon AM, Ben-Chetrit E. Clinical manifestations, risk factors and prognosis of patients with Morganella morganii sepsis. $J$ Microbiol Immunol Infect. 2019;52:443-448. doi:10.1016/j.jmii.2017.08.010

5. Huang YT, Jiang JY, Hsu MS, Hsu HS, Liao CH, Hsueh PR. The prevalence of rectal carriage of Klebsiella pneumoniae amongst diabetic patients and their clinical relevance in Taiwan: a five-year prospective study. J Microbiol Immunol Infect. 2018;51(4):510-518. doi:10.1016/j.jmii.2017.05.009

6. Du F, Wei DD, Wan LG, Cao XW, Zhang W, Liu Y. Evaluation of ompK36 allele groups on clinical characteristics and virulence features of Klebsiella pneumoniae from bacteremia. J Microbiol Immunol Infect. 2019;52(5):779-787. doi:10.1016/j.jmii.2018.08.018

7. Lee CC, Lee CH, Hong MY, Hsieh CC, Tang HJ, Ko WC. Propensity-matched analysis of the impact of extended-spectrum beta-lactamase production on adults with community-onset Escherichia coli, Klebsiella species, and Proteus mirabilis bacteremia. J Microbiol Immunol Infect. 2018;51(4):519-526. doi:10.1016/j.jmii.2017.05.006

8. Yang TY, Lu PL, Tseng SP. Update on fosfomycin-modified genes in Enterobacteriaceae. J Microbiol Immunol Infect. 2019;52(1):9-21. doi:10.1016/j.jmii.2017.10.006

9. Hung WT, Cheng MF, Tseng FC, et al. Bloodstream infection with extended-spectrum beta-lactamase-producing Escherichia coli: the role of virulence genes. J Microbiol Immunol Infect. 2019;52 (6):947-955. doi:10.1016/j.jmii.2019.03.005

10. Chen CY, Hsieh PH, Chang CY, et al. Molecular epidemiology of the emerging ceftriaxone resistant non-typhoidal Salmonella in southern Taiwan. $J$ Microbiol Immunol Infect. 2019;52(2):289-296. doi:10.1016/j.jmii.2018.08.007

11. Yang JJ, Wang JT, Cheng A, Chuang YC, Sheng WH. Impact of broad-spectrum antimicrobial treatment on the ecology of intestinal flora. J Microbiol Immunol Infect. 2018;51(5):681-687. doi:10.1016/ j.jmii.2016.12.009 
12. Martelius T, Jalava J, Karki T, et al. Nosocomial bloodstream infections caused by Escherichia coli and Klebsiella pneumoniae resistan to third-generation cephalosporins, Finland, 1999-2013: trends, patient characteristics and mortality. Infect Dis (Lond). 2016;48 (3):229-234. doi:10.3109/23744235.2015.1109135

13. Stewardson AJ, Allignol A, Beyersmann J, et al. The health and economic burden of bloodstream infections caused by antimicrobial-susceptible and non-susceptible Enterobacteriaceae and Staphylococcus aureus in European hospitals, 2010 and 2011 a multicentre retrospective cohort study. Euro Surveill. 2016;21 (33):21. doi:10.2807/1560-7917.ES.2016.21.33.30319

14. de Kraker ME, Davey PG, Grundmann H, Opal SM. Mortality and hospital stay associated with resistant Staphylococcus aureus and Escherichia coli bacteremia: estimating the burden of antibiotic resistance in Europe. PLoS Med. 2011;8(10):e1001104. doi:10.1371/ journal.pmed.1001104

15. Kang CI, Kim SH, Park WB, et al. Bloodstream infections caused by Enterobacter species: predictors of 30-day mortality rate and impact of broad-spectrum cephalosporin resistance on outcome. Clin Infect Dis. 2004;39(6):812-818. doi:10.1086/423382

16. Lin WP, Huang YS, Wang JT, Chen YC, Chang SC. Prevalence of and risk factor for community-onset third-generation cephalosporin-resistant Escherichia coli bacteremia at a medical center in Taiwan. BMC Infect Dis. 2019;19(1):245. doi:10.1186/s12879019-3880-z

17. The Clinical and Laboratory Standards InstituteI. Performance standards for antimicrobial susceptibility testing. CLSI supplement M100. 28th ed.

18. Lee CC, Lee CH, Hong MY, Tang HJ, Ko WC. Timing of appropriate empirical antimicrobial administration and outcome of adults with community-onset bacteremia. Crit Care. 2017;21(1):119. doi:10.1186/s13054-017-1696-z

19. Lee CC, Lee CH, Yang CY, Hsieh CC, Tang HJ, Ko WC. Beneficial effects of early empirical administration of appropriate antimicrobials on survival and defervescence in adults with community-onset bacteremia. Crit Care. 2019;23(1):363. doi:10.1186/s13054-0192632-1

20. David N. Selected pharmacologic features of antimicrobial agents. Sanford Guide Antimicrobial Ther. 2009;78-82.

21. Lee CC, Lee NY, Yan JJ, et al. Bacteremia due to extended-spectrum - $\beta$-lactamase-producing Enterobacter cloacae: role of carbapenem therapy. Antimicrob Agents Chemother. 2010;54(9):3551-3556. doi:10.1128/AAC.00055-10

22. Vasudevan A, Mukhopadhyay A, Li J, Yuen EG, Tambyah PA. A prediction tool for nosocomial multi-drug resistant gram-negative bacilli infections in critically ill patients - prospective observational study. BMC Infect Dis. 2014;14(1):615. doi:10.1186/s12879-0140615-z

23. Lee -C-C, Yang C-Y, Su B-A, et al. The hypotension period after initiation of appropriate antimicrobial administration is crucial for survival of bacteremia patients initially experiencing severe sepsis and septic shock. J Clin Med. 2020;9(8):2617. doi:10.3390/ jem 9082617
24. Schellevis FG, van der Velden J, van de Lisdonk E, van Eijk JT, van Weel C. Comorbidity of chronic diseases in general practice. J Clin Epidemiol. 1993;46(5):469-473. doi:10.1016/0895-4356(93)90024-U

25. Lesens O, Methlin C, Hansmann Y, et al. Role of comorbidity in mortality related to Staphylococcus aureus bacteremia: a prospective study using the Charlson weighted index of comorbidity. Infect Control Hosp Epidemiol. 2003;24:890-896.

26. Noguchi T, Matsumura Y, Yamamoto M, Nagao M, Takakura S, Ichiyama S. Clinical and microbiologic characteristics of cefotaxime-non-susceptible Enterobacteriaceae bacteremia: a case control study. BMC Infect Dis. 2017;17(1):44. doi:10.1186/s12879016-2150-6

27. Rottier WC, Bamberg YRP, Dorigo-Zetsma JW, van der Linden PD, Ammerlaan HSM, Bonten MJM. Predictive value of prior colonization and antibiotic use for third-generation cephalosporin-resistant Enterobacteriaceae bacteremia in patients with sepsis. Clin Infect Dis. 2015;60(11):1622-1630. doi:10.1093/cid/civ121

28. Chu SM, Hsu JF, Lai MY, et al. Risk factors of initial inappropriate antibiotic therapy and the impacts on outcomes of neonates with gram-negative bacteremia. Antibiotics (Basel). 2020;9.

29. Usai D, Donadu M, Bua A, et al. Enhancement of antimicrobial activity of pump inhibitors associating drugs. J Infect Dev Ctries. 2019;13(2):162-164. doi:10.3855/jidc. 11102

30. Bohnert JA, Schuster S, Fähnrich E, Trittler R, Kern WV. Altered spectrum of multidrug resistance associated with a single point mutation in the Escherichia coli RND-type MDR efflux pump YhiV (MdtF). $J$ Antimicrob Chemother. 2007;59(6):1216-1222. doi:10.1093/jac/dk1426

31. Somboro AM, Osei Sekyere J, Amoako DG, et al. In vitro potentiation of carbapenems with tannic acid against carbapenemase-producing enterobacteriaceae: exploring natural products as potential carbapenemase inhibitors. $J$ Appl Microbiol. 2019;126(2):452-467. doi:10.1111/jam.14141

32. Donadu MG, Trong Le N, Ho D V, et al. Phytochemical compositions and biological activities of essential oils from the leaves, rhizomes and whole plant of Hornstedtia bella škorničk. Antibiotics (Basel). 2020;9(6):334. doi:10.3390/antibiotics9060334

33. Le NT, Donadu MG, Ho DV, et al. Biological activities of essential oil extracted from leaves of Atalantia sessiflora Guillauminin Vietnam. J Infect Dev Ctries. 2020;14(9):1054-1064. doi:10.3855/ jidc. 12469

34. Trong Le N, Viet Ho D, Quoc Doan T, et al. Biological activities of essential oils from leaves of Paramignya trimera (Oliv.) Guillaum and Limnocitrus littoralis (Miq.) swingle. Antibiotics (Basel). 2020;9 (4):207. doi:10.3390/antibiotics9040207

35. Patterson JE, McElmeel L, Wiederhold NP. In vitro activity of essential oils against gram-positive and gram-negative clinical isolates, including carbapenem-resistant Enterobacteriaceae. Open Forum Infect Dis. 2019;6(12):ofz502. doi:10.1093/ofid/ofz502
Infection and Drug Resistance

\section{Publish your work in this journal}

Infection and Drug Resistance is an international, peer-reviewed openaccess journal that focuses on the optimal treatment of infection (bacterial, fungal and viral) and the development and institution of preventive strategies to minimize the development and spread of resistance. The journal is specifically concerned with the epidemiology of antibiotic resistance and the mechanisms of resistance development and diffusion in both hospitals and the community. The manuscript management system is completely online and includes a very quick and fair peerreview system, which is all easy to use. Visit http://www.dovepress.com/ testimonials.php to read real quotes from published authors. 